\title{
Magnitude da hipotensão pós-exercício aeróbio agudo: Uma revisão sistemática dos estudos randomizados
}

\author{
Magnitude of hypotension after acute aerobic exercise: A systematic \\ review of randomized trials
}

\author{
Ana Cristina Oliveira Marques-Silvestre, $1^{*}$ Maria do Socorro Brasileiro-Santos, ${ }^{1}$ \\ Adriana Sarmento de Oliveira, ${ }^{2}$ Fábio Thiago Maciel da Silva, ${ }^{1}$ Amilton da Cruz Santos ${ }^{1}$

\begin{abstract}
Objetivou-se revisar sistematicamente estudos randomizados sobre a magnitude da hipotensão pósexercício (HPE) em adultos. Consultaram-se as bases Pubmed, Scielo e Lilacs, combinando os indexadores "exercise" e "hypotension", sendo encontrados 1336 estudos. Destes, excluíram-se 1268 por títulos, 37 por resumos, 10 não randomizados, 1 por repetição de dados, restando 20. Estes foram avaliados segundo os itens de randomização, mascaramento, sigilo de alocação, análise por intenção de tratar e quantitativamente pela Escala de Jadad. As reduções das pressões sistólica (PAS) e diastólica (PAD) em normotensos foram inferiores à dos hipertensos $(-9 \pm 3$ vs $-11 \pm 6 \mathrm{mmHg}$ e $-5 \pm 2$ vs $-8 \pm 6$ $\mathrm{mmHg}$, respetivamente). Em ambos os grupos, os indivíduos ativos apresentaram HPE maior que os sedentários (normotensos: PAS $-10 \pm 4$ vs $-8 \pm 1 \mathrm{mmHg}$; PAD $-5 \pm 1$ vs $-4 \pm 1 \mathrm{mmHg}$; hipertensos: PAS $-16 \pm 4$ vs $-9 \pm 2 \mathrm{mmHg}$; PAD $-7 \pm 4$ vs $-5 \pm 3 \mathrm{mmHg}$, respetivamente). Nestes estudos, a magnitude da HPE variou entre $-4 \mathrm{a}-19 \mathrm{mmHg}$ para PAS e -2 a $-9 \mathrm{mmHg}$ para PAD, valores que dependem tanto das características da amostra, seus estágios pressóricos iniciais, sexo e nível de atividade física, como também do protocolo de exercício utilizado, não havendo consenso sobre a influência da sua duração ou da intensidade do exercício.

Palavras-chave: exercício, pressão arterial, hipotensão pós-exercício
\end{abstract}

ABSTRACT

The objective was to carry out a systematic review of randomized trials on the magnitude of post exercise hypotension (PEH) in adults. 1336 studies were retrieved from PubMed, Scielo and Lilacs, after searching "exercise" and "hypotension". 1268 papers were excluded by titles, 37 by abstracts, 10 were nonrandomized, 1 by repeating data, leaving 20 . These studies were evaluated according to the randomization, blinding, allocation concealment, analysis by intention to treat and quantitatively by the Jadad Scale. The average reductions in systolic (SBP) and diastolic (DBP) of the normotensive were lower than hypertensive ( $-9 \pm 3$ vs. $-11 \pm 6 \mathrm{mmHg}$ and $-12 \pm 2$ vs. $-8 \pm 6 \mathrm{mmHg}$, respectively) in both groups, active individuals had greater PEH than sedentary individuals (normotensive SBP $-10 \pm 4$ vs. $-8 \pm 1 \mathrm{mmHg}$, PAD $-5 \pm 1$ vs. $-4 \pm 1$; hypertensive SBP $-16 \pm 4$ vs $-9 \pm 2 \mathrm{mmHg}$; PAD $-7 \pm 4$ vs. $-5 \pm 3 \mathrm{mmHg}$, respectively). In randomized studies the magnitude of $\mathrm{PEH}$ ranged from -4 to -19 $\mathrm{mmHg}$ for SBP and -2 to $-9 \mathrm{~mm} \mathrm{Hg}$ for DBP values according to the sample characteristics (blood pressure early stages, gender and level of physical activity) and the exercise protocols selected (i.e., there is no consensus on the influence of duration and intensity).

Keywords: exercise, blood pressure, post-exercise hypotension

Artigo recebido a 14.05.2013; $1^{\text {a }}$ Revisão 26.03.2014; $2^{\text {a }}$ Revisão 30.06.2014; Aceite 02.07.2014

${ }^{1}$ Universidade Federal da Paraíba, Centro de Ciências da Saúde, João Pessoa, Brasil

${ }^{2}$ Instituto do Coração - HCFMUSP, Unidade de Reabilitação Cardiovascular e Fisiologia do Exercício, São Paulo, Brasil

* Autor correspondente: Universidade Federal da Paraíba, Centro de Ciências da Saúde - Campus I, Cidade Universitária - UFPB, CEP: 58059-900 João Pessoa, PB - Brasil; E-mail: acomarques@gmail.com 


\section{INTRODUÇÃO}

A hipertensão arterial sistémica (HAS) tem como aliada ao seu tratamento o exercício físico aeróbio. Esta terapêutica não farmacológica pode proporcionar benefícios importantes para os indivíduos hipertensos, não só pela redução dos níveis pressóricos, mas também pela melhoria nos diversos fatores de risco para doenças cardiovasculares (Hagberg, Park, \& Brown, 2000).

A redução da pressão arterial (PA) obtida a partir da realização de uma sessão de exercício físico é denominada de hipotensão pósexercício (HPE), e já foi observada em indivíduos hipertensos (Corazza, Gobbi, Zago, \& Costa, 2003; Cunha et al., 2006; MacDonald, MacDougall, \& Hogben, 2000; Polito, Simão, Saccomani, \& Casonatto, 2009) e normotensos (Christofaro et al., 2008; Corazza et al., 2003; MacDonald et al., 2000; Pescatello et al., 2004). Em normotensos sabe-se que uma única sessão de exercício físico aeróbio é capaz de reduzir os níveis pressóricos no momento pósexercício (Pescatello et al., 2004), enquanto, nos hipertensos, essa resposta demonstra ter maior duração (Corazza et al., 2003) e magnitude (MacDonald et al., 2000), propiciando nessa população uma terapêutica preventiva, clínica e ambulatorial para a HAS (Forjaz, Santaella, Rezende, Barretto, \& Negrão, 1998; Forjaz et al., 2000; Lizardo, Modesto, Campbell, \& Simões, 2007).

Diversas revisões já foram realizadas sobre o efeito hipotensor do exercício aeróbio abordando principalmente os mecanismos envolvidos e as diversas variáveis que podem influenciar a sua resposta (Cardoso Jr et al., 2010; Casonatto \& Polito, 2009; Hagberg et al., 2000; Kenney \& Seals, 1993; MacDonald et al., 2000). Embora o fenómeno da HPE em indivíduos hipertensos esteja bem estabelecido, acreditamos ser necessário termos um consenso sobre os valores dessa resposta aguda. Assim, a verificação da magnitude da HPE em estudos randomizados pode auxiliar no estabelecimento destes valores. Sendo assim, o objetivo dessa pesquisa é revisar sistematicamente estudos randomizados que abordaram a magnitude da diminuição pressórica em adultos, verificando esses valores após uma única sessão de exercício aeróbio.

\section{MÉTODO}

A busca dos artigos foi realizada sistematicamente até junho de 2014, nas bases de dados PubMed, Scielo e Lilacs, utilizando-se os indexadores de forma combinada "exercise" e "hypotension", ambos especificados nos Descritores de Ciências da Saúde (DeCS), não havendo restrição de idiomas.

Posteriormente, os artigos foram selecionados de forma independente, inicialmente pelo título e em seguida pelo resumo e texto completo, por dois pesquisadores experientes. Foram incluídos apenas estudos randomizados, realizados em humanos com idade superior a 19 anos e que abordassem a magnitude da HPE após a realização de exercício aeróbio agudo. No caso de divergência de opiniões, um terceiro avaliador foi consultado para solucioná-la. Excluiu-se desta pesquisa artigos de revisão de literatura ou que estivessem repetidos nas bases consultadas, como também, os estudos abordando exercício resistido, isométrico e crónico; e, estudos com animais experimentais, atletas, gestantes, crianças e adolescentes.

Nos artigos que abordavam mais de um protocolo de exercício, avaliaram-se apenas as informações obtidas a partir do protocolo de exercício aeróbio contínuo e de intensidade leve, moderada e intensa.

A avaliação metodológica dos artigos selecionados foi realizada com base nos componentes individuais de qualidade (Rech, Nóbrega, \& Lemos, 2010) que inclui os itens: randomização, mascaramento, sigilo de alocação e análise por intenção de tratar. Em cada um destes itens foi atribuído um dos seguintes escores: adequado, inadequado, não realizado ou não referido. Adicionalmente, houve a avaliação quantitativa da qualidade dos artigos através do uso da Escala de Jadad (Jadad et al., 1996). O sistema de Jadad consta de tópicos centrados na validade interna da pesquisa refe- 
rentes à randomização, mascaramento duplocego, perda e exclusão de participantes, diretamente relacionadas com a redução de vieses. Para cada resposta "sim" é atribuído um ponto na classificação, e um ponto adicional se o método de randomização e mascaramento forem descritos de forma adequada. Caso sejam citados de forma inadequada, deve-se reduzir um ponto. $\mathrm{O}$ máximo de pontos que pode ser obtido na escala são 5 , sendo considerado de boa qualidade aquele que receber mais de 2 pontos. Para a inclusão dos estudos nessa revisão, os artigos escolhidos deveriam possuir, no mínimo, pontuação referente ao critério de randomização.

\section{RESULTADOS}

Como resultado da busca combinada dos termos indexadores "exercise" and "hypotension" nas bases de dados, foram catalogados 99 arti- gos no Lilacs, 40 no Scielo, e 1197 no Pubmed, totalizando 1336 artigos. Destes, foram excluídos inicialmente 1268 por título, uma vez que os mesmos não correspondiam aos nossos critérios de inclusão. Posteriormente, dos 68 artigos selecionados, 37 deles foram excluídos por diversos aspetos: 11 estavam com resumos indisponíveis nas bases de dados; 2 se tratavam de exercício resistido; 1 com amostra animal; 14 por não abordarem especificamente sobre a HPE; 2 realizaram exercício crônico e 2 exercício combinado; 3 eram revisões sistemáticas; 2 tratavam da HPE mas, com suplementação de substâncias ou alimentos. Por fim, dos 31 resumos restantes, foram obtidos seus textos completos e analisados, sendo selecionados apenas aqueles randomizados, totalizando no final 21 estudos. No entanto, durante a tabulação dos dados, observou-se a repetição de resultados de dois estudos que possuíam o

\begin{tabular}{|c|c|}
\hline \multirow{3}{*}{$\begin{array}{l}\text { Artigos relevantes selecionados nas bases de } \\
\text { dados com os indexadores combinados: } \\
\text { "exercise" e "hypotension" ( } \mathrm{n} \text { total }=1336 \text { ) } \\
\text { Lilacs } n=99 ; \text { Scielo } n=40 ; \text { Pubmed } n=1197\end{array}$} & $\begin{array}{l}\text { Artigos excluídos após a análise } \\
\text { dos títulos }(n=1268)\end{array}$ \\
\hline & $\begin{array}{l}\text { (Repetido nas bases de dados; exercício resistido, } \\
\text { isométrico, crónico; com animais, atletas, gestantes, }\end{array}$ \\
\hline & $\begin{array}{c}\text { crianças, adolescentes; revisões; não tratam sobre a } \\
\text { magnitude da HPE) }\end{array}$ \\
\hline
\end{tabular}

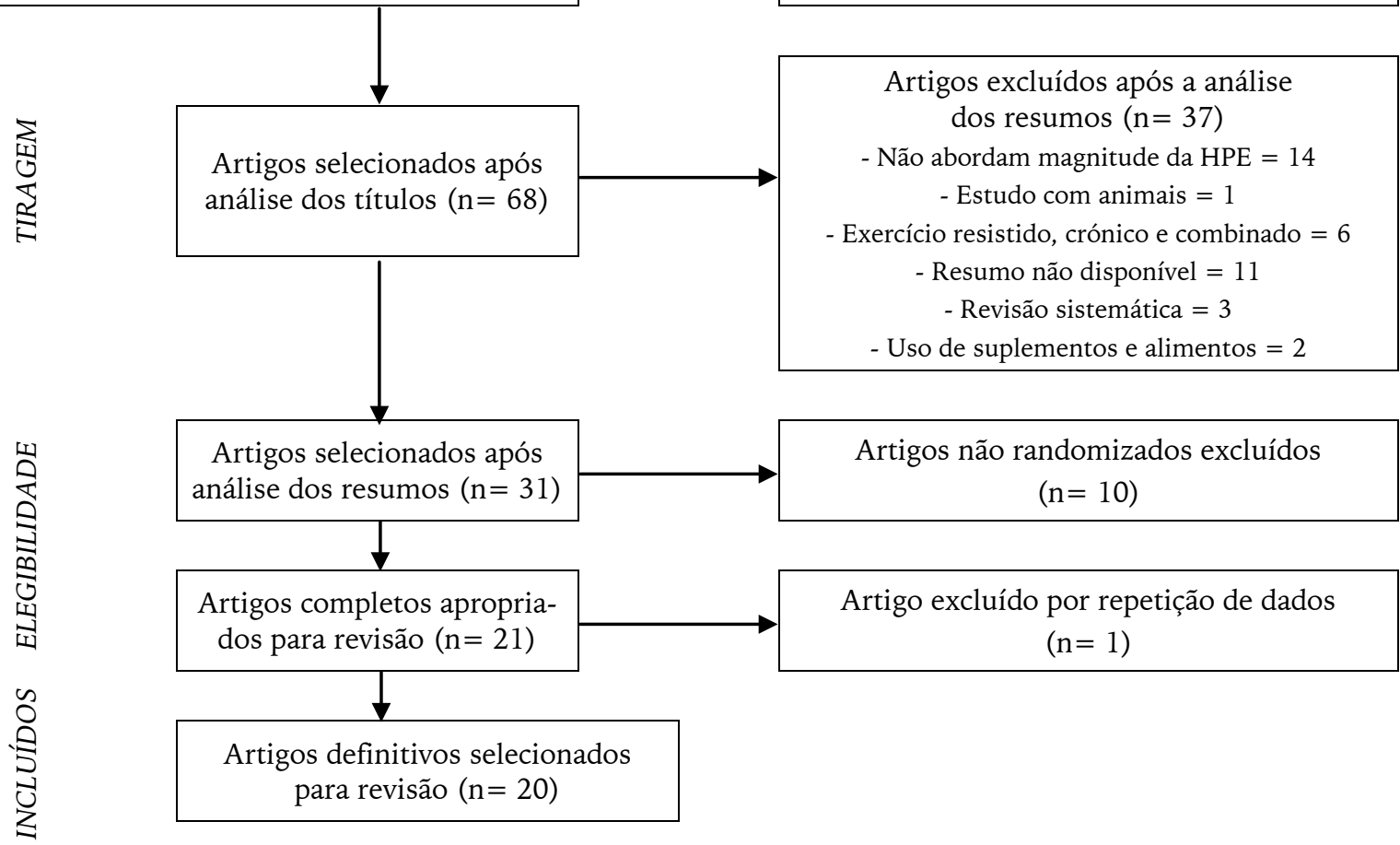

Figura 1. Busca e seleção dos artigos para revisão sistemática 
mesmo autor principal (Ferreira, Campos, Rodrigues Junior, Puga, \& Brito, 2013; Ferreira, Ferreira, Campos, Samy, \& de Morais, 2011), visto que eles se tratavam da mesma amostra do protocolo aeróbio. Estes artigos se diferenciavam apenas por um deles ter o acréscimo do protocolo de exercício resistido, sendo desta forma, considerado apenas o artigo mais recente (Ferreira et al., 2013), restando assim, 20 estudos (Figura 1 ).

Dos 20 artigos selecionados, um deles (Casonatto, Tinucci, Dourado, \& Polito, 2011) citou o método utilizado para a organização da sequência de randomização de forma adequa- da. Apenas um estudo (Pescatello et al., 2004) citou a execução do mascaramento, mas não referiu como este foi realizado. O mesmo ocorreu para o sigilo de alocação, citado por dois estudos (Casonatto et al., 2011; Pescatello et al., 2004), mas não foi possível verificar a sua adequação. A análise por intenção de tratar não foi realizada pelos estudos (Tabela 1), no entanto, justificamos este fato por serem pesquisas cuja intervenção com exercício foi feita de forma aguda e não crónica.

De acordo com a Escala de Jadad, todos os 20 artigos selecionados são randomizados, e um deles expôs seu método de randomização

Tabela 1

Avaliação da qualidade metodológica dos artigos selecionados

\begin{tabular}{|c|c|c|c|c|}
\hline Autor (Ano) & Randomização & Mascaramento & $\begin{array}{l}\text { Sigilo de } \\
\text { Alocação }\end{array}$ & $\begin{array}{c}\text { Análise por } \\
\text { intenção de tratar }\end{array}$ \\
\hline Cal Abad et al. (2010) & não referido & não realizado & não realizado & não realizado \\
\hline Casonatto et al. (2011) & adequado & não realizado & não realizado & não realizado \\
\hline Christofaro et al. (2008) & não referido & não realizado & não realizado & não realizado \\
\hline Cunha et al. (2006) & não referido & não realizado & não realizado & não realizado \\
\hline Ferreira et al. (2013) & não referido & não realizado & não realizado & não realizado \\
\hline Forjaz et al. (2004) & não referido & não realizado & não realizado & não realizado \\
\hline $\begin{array}{l}\text { Forjaz, Matsudaira, et al. } \\
\text { (1998) }\end{array}$ & não referido & não realizado & não realizado & não realizado \\
\hline Forjaz, Santaella, et al. (1998) & não referido & não realizado & não realizado & não realizado \\
\hline Forjaz et al. (2000) & não referido & não realizado & não realizado & não realizado \\
\hline $\begin{array}{c}\text { Jones, George, Edwards e } \\
\text { Atkinson (2007) }\end{array}$ & não referido & não realizado & não realizado & não realizado \\
\hline Lizardo et al. (2007) & não referido & não realizado & não realizado & não realizado \\
\hline $\begin{array}{l}\text { MacDonald, MacDougall e } \\
\text { Hogben (1999) }\end{array}$ & não referido & não realizado & não realizado & não realizado \\
\hline MacDonald et al. (2000) & não referido & não realizado & não realizado & não realizado \\
\hline Mota et al. (2009) & não referido & não realizado & não realizado & não realizado \\
\hline Pescatello et al. (1999) & não referido & não realizado & não realizado & não realizado \\
\hline Pescatello et al. (2003) & não referido & não realizado & não realizado & não realizado \\
\hline Pescatello et al. (2004) & não referido & não referido & não referido & não realizado \\
\hline Rossow et al. (2010) & não referido & não realizado & não realizado & não realizado \\
\hline Santana et al. (2011) & não referido & não realizado & não realizado & não realizado \\
\hline Santana et al. (2013) & não referido & não realizado & não realizado & não realizado \\
\hline
\end{tabular}


Tabela 2

Avaliação dos artigos selecionados nessa revisão sistemática de acordo com a Escala de Jadad et al. (1996)

\begin{tabular}{|c|c|c|c|}
\hline \multirow{2}{*}{ Itens da Escala de Jadad } & \multicolumn{2}{|c|}{ Pontuação para respostas } & \multirow{2}{*}{$\mathrm{N}$} \\
\hline & $\operatorname{Sim}$ & Não & \\
\hline \multicolumn{4}{|l|}{ Randomização } \\
\hline O estudo foi descrito como randomizado? & 1 & 0 & 20 \\
\hline Descreve o método de randomização? & 1 & 0 & 1 \\
\hline O método de randomização é adequado? & 1 & -1 & 1 \\
\hline \multicolumn{4}{|l|}{ Mascaramento } \\
\hline O estudo foi descrito como duplo-cego? & 1 & 0 & 1 \\
\hline Descreve o método de mascaramento? & 1 & 0 & $0^{*}$ \\
\hline O método de mascaramento é adequado? & 1 & -1 & - \\
\hline \multicolumn{4}{|l|}{ Perdas e retiradas } \\
\hline Houve descrição de perdas e retiradas? & 1 & 0 & 1 \\
\hline
\end{tabular}

de forma adequada (Casonatto et al., 2011). Apenas 1 estudo era duplo-cego (Pescatello et al., 2004), mas não descreveu seu método de mascaramento. Um estudo (Santana et al., 2011) citou se houve perdas e retiradas (tabela 2). Foi possível observar ainda que 14 dos 20 artigos selecionados são randomizadoscontrolados (Casonatto et al., 2011; Christofaro et al., 2008; Ferreira et al., 2013; Forjaz et al., 2000, 2004; Forjaz, Matsudaira, et al., 1998; Forjaz, Santaella, et al., 1998; Jones et al., 2007; Mota et al., 2009; Pescatello et al., 1999, 2003, 2004; Santana et al., 2011, 2013).

Neste estudo, a randomização foi um critério de entrada para seleção dos artigos elegíveis, porém, a maioria não citou quais os métodos utilizados, não sendo possível verificá-los quanto a sua adequação. Resultado similar foi observado numa revisão com mais de 80 relatórios de ensaios clínicos randomizados encontrados em quatro importantes revistas da área médica. Nessa foi observado que o relato da metodologia de randomização foi sempre inadequado (Altman \& Dore, 1990). Verificouse num estudo (Moher et al., 2010) que, nos ensaios indexados no PubMed em 2006, o método para a randomização predominante foi a utilização de um gerador de números aleató- rios num computador ou numa tabela de números aleatórios.

Uma avaliação metodológica de 519 ensaios indexados no PubMed e publicados em dezembro de 2000 , demonstrou que $60 \%$ deles eram "cegos" e que o poder de cálculo, resultados primários, geração de sequência aleatória e o sigilo da alocação de cada um deles só foram adequadamente descritos em menos de 50\% (Chan \& Altman, 2005). De todos os ensaios clínicos randomizados indexados no PubMed, apenas $18 \%$ deles relataram algum mecanismo de sigilo de alocação, sendo alguns deles inadequados (Moher et al., 2010).

A randomização tem a vantagem de que quando devidamente implementada eliminar o viés de seleção na atribuição dos tratamentos (Moher et al., 2010). Sendo assim, a avaliação da qualidade dos ensaios clínicos randomizados tem grande importância, visto que nos fornece uma probabilidade de que os resultados seja uma estimativa válida da verdade (Moher et al., 1995). Por isso, independente de outros fatores avaliados, a randomização foi o critério principal exigido para a inclusão dos artigos nesta revisão, visto que, pela natureza da intervenção do exercício, ela pode ser de grande importância na eliminação de vieses. 
Assim como a sessão controle, cuja maioria dos estudos, no total de $70 \%$, a citou (Christofaro et al., 2008; Forjaz et al., 2000, 2004; Forjaz, Matsudaira, et al., 1998; Forjaz, Santaella, et al., 1998; Lizardo et al., 2007; Mota et al., 2009; Pescatello et al., 1999, 2003, 2004). Incluímos os estudos não-controlados e também com ausência dos outros critérios de avaliação a fim de aumentar a possibilidade de comparação dos resultados. Além disso, esta realidade foi semelhante à encontrada em outros estudos realizados a respeito da qualidade metodológica dos artigos publicados na área médica (Altman \& Dore, 1990; Chan \& Altman, 2005; Moher et al., 2010).

A magnitude da HPE foi obtida calculando a diferença dos valores de pressão obtidos entre a condição pós-exercício e pré-exercício (Cal Abad, da Silva, Mostarda, da Silva, \& Irigoyen, 2010; Casonatto et al., 2011; Christofaro et al., 2008; Cunha et al., 2006; Forjaz et al., 2004; Forjaz, Matsudaira, et al., 1998; Forjaz, Santaella, et al., 1998; Lizardo et al., 2007; MacDonald, MacDougall, \& Hogben, 1999; MacDonald et al., 2000; Mota et al., 2009; Pescatello et al., 1999, 2003, 2004; Rossow et al., 2010; Santana et al., 2011, 2013) ou entre a sessão exercício e a sessão controle (Ferreira et al., 2013; Forjaz et al., 2000; Jones et al., 2007), citadas como estatisticamente significante nos artigos $(p \leq 0.05)$.

\section{DISCUSSÃO}

Os momentos pós-exercícios verificados foram distintos nos vários estudos. Um deles avaliou a HPE até 15 minutos (Cal Abad et al., 2010) e outro até 20 minutos após o exercício (Jones et al., 2007). Outros avaliaram até 60 minutos (Casonatto et al., 2011; Christofaro et al., 2008; MacDonald et al., 1999, 2000; Rossow et al., 2010; Santana et al., 2011, 2013), até 90 minutos (Forjaz, Matsudaira, et al., 1998; Forjaz, Santaella, et al., 1998; Lizardo et al., 2007) e até 120 minutos (Cunha et al., 2006) após o exercício. Outros estudos verificaram a resposta ambulatorial da HPE, observando essa em até 7 horas (Mota et al., 2009;
Pescatello et al., 1999) ou em 24 horas (Ferreira et al., 2013; Forjaz et al., 2000, 2004; Pescatello et al., 2003, 2004). Neste último, algumas pesquisas apresentaram seus resultados de forma que o período de 24 horas foi dividido em day time e nigth time ou a seja, as médias das reduções da PA foram obtidas nos momentos diurnos e noturnos, respetivamente.

Todos os estudos aferiram a PA em situação basal com métodos validados, sendo eles auscultatório, oscilométrico ou intravenoso, após manter os indivíduos em repouso durante 5 (Forjaz et al., 2000), 10 (Casonatto et al., 2011; Cunha et al., 2006; Rossow et al., 2010), 15 (Cal Abad et al., 2010; Christofaro et al., 2008; Ferreira et al., 2013; Pescatello et al., 1999, 2003), 20 (Forjaz et al., 2004; Forjaz, Matsudaira, et al., 1998; Forjaz, Santaella, et al., 1998; Lizardo et al., 2007; Mota et al., 2009; Pescatello et al., 2004) e 30 minutos (MacDonald et al., 1999, 2000).

A média \pm desvio-padrão das magnitudes das quedas pressóricas sistólicas, diastólicas e médias obtidas nos estudos selecionados foram $-9.4 \pm 4.2,-5.0 \pm 2.1$ e $-6.2 \pm 3.2 \mathrm{mmHg}$, respetivamente, considerando os protocolos e tempos de recuperação para ocorrência da HPE que obtiveram maiores reduções da pressão arterial e que a expressou em dados numéricos.

Nos artigos selecionados (Tabela 3), as maiores quedas pressóricas observadas foram para pressão sistólica de $-19 \mathrm{mmHg}$, diastólica $-9.0 \mathrm{mmHg}$ e média $-13 \mathrm{mmHg}$, cuja amostra era de indivíduos hipertensos (Cunha et al., 2006). Observou-se de uma forma geral que, nos normotensos, o cálculo das médias das magnitudes da HPE sistólica e da HPE média foi menor do que nos hipertensos $(-9 \pm 3$ vs. $-11 \pm 6 \mathrm{mmHg}$ e $-5 \pm 2$ vs. $-8 \pm 6 \mathrm{mmHg}$, respetivamente), e a magnitude da HPE diastólica foi semelhante $(-5 \pm 2 v s-5 \pm 3 \mathrm{mmHg})$.

Os momentos de observação da PA pósexercício que registraram valores significativos da HPE nos diferentes protocolos de exercício físico foram de 5, 15, 30, 45, 60, 75, 90 minutos, 9 horas e nigth-time. A maior incidência de registros das quedas sistólicas (Lizardo et al., 
Tabela 3

Características gerais dos estudos selecionados e suas magnitudes das reduções pressóricas sistólicas, diastólicas e médias (mmHg) obtidas após uma sessão de exercício aeróbio agudo

\begin{tabular}{|c|c|c|c|c|c|c|c|}
\hline \multirow[t]{2}{*}{ Autores (ano) } & \multirow{2}{*}{$\begin{array}{c}\mathrm{n} \\
(\mathrm{H} / \mathrm{M})\end{array}$} & \multirow{2}{*}{$\begin{array}{l}\text { Idade (anos)/ } \\
\text { Amostra }\end{array}$} & \multirow{2}{*}{$\begin{array}{l}\text { Intensidade/ } \\
\text { duração do EF }\end{array}$} & \multirow{2}{*}{$\begin{array}{l}\text { Valores basais } \\
\text { PAS/PSD/PAM } \\
\quad(\mathrm{mmhg})\end{array}$} & \multicolumn{3}{|c|}{$\begin{array}{l}\text { Tempo para ocorrência pós EF (min ou hora) e } \\
\text { magnitude da HPE }\end{array}$} \\
\hline & & & & & Sistólica & Diastólica & Média \\
\hline Cal Abad et al., (2010) & $\begin{array}{c}10 \\
(10 / 0)\end{array}$ & $\begin{array}{l}23 \pm 4 \\
\text { NT/ A }\end{array}$ & 60 a $70 \%$ FC res/30min & NI & $15: \rightarrow$ & $15: \rightarrow$ & $15: \rightarrow$ \\
\hline Casonatto et al. (2011) & $\begin{array}{c}15 \\
(15 / 0)\end{array}$ & $\begin{array}{l}25 \pm 1 \\
\text { NT/ A }\end{array}$ & $\begin{array}{c}80 \% \mathrm{VO}_{2} \mathrm{pico} / 30 \mathrm{~min} \\
60 \% \mathrm{VO}_{2} \mathrm{pico} / \sim 45 \mathrm{~min} \\
60 \% \mathrm{VO}_{2} \mathrm{pico} / 30 \mathrm{~min} \\
40 \% \mathrm{VO}_{2} \mathrm{pico} / \sim 60 \mathrm{~min}\end{array}$ & $\begin{array}{l}114 / 67 / \mathrm{NI} \\
113 / 67 / \mathrm{NI} \\
115 / 67 / \mathrm{NI} \\
115 / 66 / \mathrm{NI}\end{array}$ & $\begin{array}{l}5 \text { a } 60: \rightarrow \\
5 \text { a } 60: \rightarrow \\
5 \text { a } 60: \rightarrow \\
5 \text { a } 60: \rightarrow\end{array}$ & $\begin{array}{c}5:+7 ; 10 \text { a } 60: \rightarrow^{\S} \\
5 \text { a } 60: \rightarrow^{\S} \\
5 \text { a } 60: \rightarrow^{\S} \\
5 \text { a } 60: \rightarrow^{\S}\end{array}$ & $\begin{array}{l}\mathrm{NI} \\
\mathrm{NI} \\
\mathrm{NI} \\
\mathrm{NI}\end{array}$ \\
\hline Christofaro et al. (2008) & $\begin{array}{c}36 \\
(36 / 0)\end{array}$ & $\begin{array}{l}29 \pm 5 \\
\mathrm{NT} / \mathrm{S}\end{array}$ & $\begin{array}{l}75 \% \text { FC máx/20 min } \\
75 \% \text { FC máx /40 min }\end{array}$ & $\begin{array}{l}120 / 83 / \mathrm{NI} \\
126 / 86 / \mathrm{NI}\end{array}$ & $\begin{array}{l}60:-4 \\
60:-8\end{array}$ & $\rightarrow$ & NI \\
\hline Cunha et al. (2006) & $\begin{array}{l}11 \\
\mathrm{NI}\end{array}$ & $\begin{array}{l}57 \pm 3 \\
\mathrm{HT} / \mathrm{A}\end{array}$ & $\begin{array}{c}60 \% \mathrm{FC} \mathrm{res} / 45 \mathrm{~min} \\
50 \text { e } 80 \% \mathrm{FC} \text { res } / 45 \mathrm{~min}\end{array}$ & NI & $\begin{array}{l}5 \text { a } 60:-19 \\
5 \text { a } 60:-18\end{array}$ & $\begin{aligned} 30: & -9 \\
& \rightarrow\end{aligned}$ & $\begin{array}{c}30:-13 \\
5.15 \text { e } 60:-10\end{array}$ \\
\hline Ferreira et al. (2013) & $\begin{array}{c}24 \\
(0 / 24)\end{array}$ & $\begin{array}{l}33 \pm 9 \\
\mathrm{NT} / \mathrm{S}\end{array}$ & $\begin{array}{c}50 \% \text { FC res/ > } 30 \mathrm{~min} \\
70 \% \text { FC res/ } 30 \mathrm{~min}\end{array}$ & $116 / 79 / 92$ & $\begin{array}{l}45 \text { a } 24 \text { hs: } \downarrow^{\dagger \$} \\
45 \text { a } 24 \text { hs: } \downarrow^{\dagger \$}\end{array}$ & $\begin{array}{l}30 \text { a } 7 \text { hs: } \downarrow^{\dagger s} \\
30 \text { a } 24 \text { hs: } \downarrow^{\dagger \S}\end{array}$ & $\begin{array}{l}\mathrm{NI} \\
\mathrm{NI}\end{array}$ \\
\hline Forjaz et al. (2004) & $\begin{array}{c}23 \\
(8 / 15)\end{array}$ & $\begin{array}{l}24 \pm 1 \\
\mathrm{NT} / \mathrm{S}\end{array}$ & $\begin{array}{l}30 \% \mathrm{VO}_{2} \mathrm{pico} / 45 \mathrm{~min} \\
50 \% \mathrm{VO}_{2} \mathrm{pico} / 45 \mathrm{~min} \\
75 \% \mathrm{VO}_{2} \mathrm{pico} / 45 \mathrm{~min}\end{array}$ & $109 / 71 / 84$ & $\begin{array}{c}\rightarrow \\
60:-7^{\dagger} \\
60:-9^{\dagger}\end{array}$ & $\begin{array}{c}\rightarrow \\
30:-4^{\dagger} \\
60:-4^{\dagger}\end{array}$ & $\begin{array}{l}30 \text { e } 60:-4 \\
60:-6\end{array}$ \\
\hline $\begin{array}{l}\text { Forjaz, Matsudaira, et al. } \\
\text { (1998) }\end{array}$ & $\begin{array}{c}24 \\
(13 / 11)\end{array}$ & $\begin{array}{l}22 \pm 1 \\
\mathrm{NT} / \mathrm{S}\end{array}$ & $\begin{array}{l}30 \% \mathrm{VO}_{2} \mathrm{pico} / 45 \mathrm{~min} \\
50 \% \mathrm{VO}_{2} \mathrm{pico} / 45 \mathrm{~min} \\
80 \% \mathrm{VO}_{2} \mathrm{pico} / 45 \mathrm{~min}\end{array}$ & $\begin{array}{l}110 / 76 / 87 \\
107 / 75 / 86 \\
105 / 73 / 84\end{array}$ & $\begin{array}{l}3 \text { a } 30:-4^{\S} \\
35 \text { a } 60:-6^{\S} \\
65 \text { a } 90:-5^{\S}\end{array}$ & $\begin{array}{l}5 \text { a } 30:-3^{\S} \\
35 \text { a } 60:-4^{\S} \\
65 \text { a } 90:-3^{\S}\end{array}$ & $\begin{array}{l}5 \text { a } 30:-3^{\S} \\
35 \text { a } 60:-5^{\S} \\
65 \text { a } 90:-3^{\S}\end{array}$ \\
\hline $\begin{array}{l}\text { Forjaz, Santaella, et al. } \\
\text { (1998) }\end{array}$ & $\begin{array}{c}22 \\
(10 / 12)\end{array}$ & $\begin{array}{l}22 \pm 1 \\
\mathrm{NT} / \mathrm{S}\end{array}$ & $\begin{array}{l}50 \% \mathrm{VO}_{2} \mathrm{pico} / 25 \mathrm{~min} \\
50 \% \mathrm{VO}_{2} \mathrm{pico} / 45 \mathrm{~min}\end{array}$ & $\begin{array}{l}110 / 76 / 87 \\
111 / 76 / 87\end{array}$ & $\begin{array}{c}75:-7 \\
30 \text { e } 90:-8\end{array}$ & $\begin{array}{l}45: \downarrow \\
45: \downarrow\end{array}$ & $\begin{array}{l}\text { 45: } \downarrow \\
45: \downarrow\end{array}$ \\
\hline Forjaz et al. (2000) & $\begin{array}{c}53 \\
(31 / 22)\end{array}$ & $\begin{array}{c}35 \pm 2 \\
\text { NT e HT } 1 / \mathrm{S}\end{array}$ & $50 \% \mathrm{VO}_{2} \mathrm{pico} / 45 \mathrm{~min}$ & $\mathrm{NI}$ & $\begin{array}{l}\mathrm{HT}: \rightarrow \\
\mathrm{NT}: \rightarrow\end{array}$ & $\begin{array}{c}\text { HT: } \rightarrow \\
\text { Nigth: NT: }-2^{\dagger}\end{array}$ & $\begin{array}{c}\text { HT: } \rightarrow \\
\text { Nigth: NT: }-2^{\dagger}\end{array}$ \\
\hline Jones et al. (2007) & $\begin{array}{c}7 \\
(7 / 0)\end{array}$ & $\begin{array}{l}28 \pm 6 \\
\text { NT/ A }\end{array}$ & $\begin{array}{c}70 \% \mathrm{VO}_{2} \mathrm{pico} / 30 \mathrm{~min} \\
40 \% \mathrm{VO}_{2} \mathrm{pico} / 30 \mathrm{~min} \\
40 \% \mathrm{VO}_{2} \mathrm{pico} / 50.0 \pm 8.0 \mathrm{~min}\end{array}$ & NI & $\begin{array}{c}20:-15^{\dagger} \\
20:-6^{\dagger} \\
20:-12^{\dagger}\end{array}$ & $\begin{array}{l}20:-5^{\dagger} \\
20:-6^{\dagger} \\
20:-5^{\dagger}\end{array}$ & $\begin{array}{l}20:-5^{\dagger} \\
20:-6^{\dagger} \\
20:-5^{\dagger}\end{array}$ \\
\hline Lizardo et al. (2007) & $\begin{array}{c}10 \\
(10 / 0)\end{array}$ & $\begin{array}{l}25 \pm 4 \\
\text { NT/ A }\end{array}$ & $\begin{array}{c}\text { Esteira, } 85 \% \text { FC máx/20 min } \\
\text { Cicloergómetro, } 85 \% \text { da } \\
\text { FC máx/20 min }\end{array}$ & NI & $\begin{array}{c}45 \text { e } 90: \downarrow \\
90: \downarrow\end{array}$ & $\begin{array}{c}0 \text { a } 90: \downarrow \\
90: \downarrow\end{array}$ & NI \\
\hline
\end{tabular}


Tabela 3 (cont.)

Características gerais dos estudos selecionados e suas magnitudes das reduções pressóricas sistólicas, diastólicas e médias (mmHg) obtidas após uma sessão de exercício aeróbio agudo

\begin{tabular}{|c|c|c|c|c|c|c|c|}
\hline \multirow{2}{*}{ Autores (ano) } & \multirow{2}{*}{$\begin{array}{c}\mathrm{n} \\
(\mathrm{H} / \mathrm{M})\end{array}$} & \multirow{2}{*}{$\begin{array}{l}\text { Idade (anos)/ } \\
\text { Amostra }\end{array}$} & \multirow{2}{*}{$\begin{array}{l}\text { Intensidade/ } \\
\text { duração do EF }\end{array}$} & \multirow{2}{*}{$\begin{array}{l}\text { Valores basais } \\
\text { PAS/PSD/PAM } \\
\text { (mmh) }\end{array}$} & \multicolumn{3}{|c|}{$\begin{array}{c}\text { Tempo para ocorrência pós EF (min ou hora) e } \\
\text { magnitude da HPE }\end{array}$} \\
\hline & & & & & Sistólica & Diastólica & Média \\
\hline MacDonald et al. (1999) & $\begin{array}{c}10 \\
(6 / 4)\end{array}$ & $\begin{array}{l}35 \pm 16 \\
\text { NT/ A }\end{array}$ & 50 e $75 \% \mathrm{VO}_{2} \mathrm{pico} / 30 \mathrm{~min}$ & $132 / 75 / 93$ & $5:-8^{\Im}$ & $30:-5^{s}$ & $15:-6^{\Im}$ \\
\hline MacDonald et al. (2000) & $\begin{array}{c}21 \\
(19 / 2)\end{array}$ & $\begin{array}{c}23 \pm 2 \\
\text { NT e LT/ A }\end{array}$ & $\begin{array}{c}70 \% \mathrm{VO}_{2} \mathrm{pico} / 15,30 \text { e } 45 \mathrm{~min} \\
70 \% \mathrm{VO}_{2} \mathrm{pico} / 10 \text { e } 30 \mathrm{~min}\end{array}$ & $\begin{array}{l}126 / 71 / 86 \\
133 / 79 / 96\end{array}$ & $\begin{array}{l}45:-12^{\Im} \\
15:-14^{\Im}\end{array}$ & $\begin{array}{l}45:-5 \sqrt{ } \\
15:-8\end{array}$ & $\begin{array}{l}45:-7^{\S} \\
15:-10^{\S}\end{array}$ \\
\hline Mota et al. (2009) & $\begin{array}{c}15 \\
(13 / 2)\end{array}$ & $\begin{array}{r}43 \pm 2 \\
\mathrm{HT} 1 / \mathrm{A}\end{array}$ & 70 a $80 \%$ FCres $/ 20 \mathrm{~min}$ & $134 / 85 / \mathrm{NI}$ & 30: -11 & $45:-4$ & $\downarrow$ \\
\hline Pescatello et al. (1999) & $\begin{array}{c}18 \\
(0 / 18)\end{array}$ & $\begin{array}{c}36 \pm 2 \\
\text { NT e HT/ NI }\end{array}$ & $60 \% \mathrm{VO}_{2} \mathrm{pico} / 40 \mathrm{~min}$ & $\begin{array}{l}\text { HT: } 143 / 96 / 112 \\
\text { NT: } 110 / 73 / 86\end{array}$ & $\begin{array}{l}\text { 7h: HT: }-10 \\
\text { 7h: NT: } \rightarrow\end{array}$ & $\begin{array}{l}\text { 7h: HT: }-7 \\
\text { 7h: NT: } \rightarrow\end{array}$ & $\begin{array}{l}\text { 7h: HT: }-8 \\
\text { 7h: NT: } \rightarrow\end{array}$ \\
\hline Pescatello et al. (2003) & $\begin{array}{c}33 \\
(0 / 33)\end{array}$ & $\begin{array}{c}31 \pm 2 \\
\text { NT e HT } 1 / \mathrm{S}\end{array}$ & $60 \% \mathrm{VO}_{2} \mathrm{pico} / 30 \mathrm{~min}$ & $\begin{array}{l}\text { HT b: } 143 / 96 / \mathrm{NI} \\
\text { HT n: } 122 / 81 / \mathrm{NI} \\
\text { NT b: } 111 / 73 / \mathrm{NI} \\
\text { NT n: } 104 / 69 / \mathrm{NI}\end{array}$ & $\begin{array}{l}\text { Day: HT b: }-11 \\
\text { Day: HT n: }+13 \\
\text { Day: NT b: } \rightarrow \\
\text { Day: NT n: }+6\end{array}$ & $\begin{array}{l}\text { Day: HT b: }-8 \\
\text { Day: HT n: } \rightarrow \\
\text { Day: NT b: } \rightarrow \\
\text { Day: NT n: } \rightarrow\end{array}$ & NI \\
\hline Pescatello et al. (2004) & $\begin{array}{c}49 \\
(49 / 0)\end{array}$ & $\begin{array}{l}44 \pm 1 \\
\mathrm{HT} 1 / \mathrm{S}\end{array}$ & $\begin{array}{l}40 \% \mathrm{VO}_{2} \mathrm{máx} / 40 \mathrm{~min} \\
60 \% \mathrm{VO}_{2} \mathrm{máx} / 40 \mathrm{~min}\end{array}$ & $126 / 87 / \mathrm{NI}$ & $\begin{array}{l}9 \mathrm{~h}:+8 \\
9 \mathrm{~h}:+7\end{array}$ & $\begin{array}{l}9 \mathrm{~h}:-3 \\
9 \mathrm{~h}:-3\end{array}$ & NI \\
\hline Rossow et al. (2010) & $\begin{array}{c}25 \\
(15 / 10)\end{array}$ & $\begin{array}{l}26 \pm 1 \\
\mathrm{NT} / \mathrm{A}\end{array}$ & $60 \%$ FCres/60 min & $116 / 63 / 74$ & $60:-5$ & $60:-4$ & $60:-4$ \\
\hline Santana et al. (2011) & $\begin{array}{c}30 \\
(0 / 30)\end{array}$ & $\begin{array}{l}70 \pm 6 \\
\mathrm{HT} / \mathrm{NI}\end{array}$ & $90 \% \mathrm{LA} / 20 \mathrm{~min}$ & $\begin{array}{l}\text { II/ID: } 123 / 75 / 91 \\
\text { DD: } 117 / 72 / 87\end{array}$ & $\begin{array}{l}60:-4^{\dagger} \\
60:+2^{*}\end{array}$ & $\begin{array}{l}60: \rightarrow \\
60: \rightarrow\end{array}$ & $\begin{array}{c}60:-1 \dagger \\
60: \rightarrow\end{array}$ \\
\hline Santana et al. (2013) & $\begin{array}{c}23 \\
(0 / 23)\end{array}$ & $\begin{array}{l}70 \pm 6 \\
\mathrm{HT} / \mathrm{NI}\end{array}$ & $90 \% \mathrm{LA} / 20 \mathrm{~min}$ & $121 / 74 / 90$ & $60: \rightarrow$ & $60: \rightarrow$ & $60: \rightarrow$ \\
\hline
\end{tabular}

Nota: H - homem; M - mulher; PA - pressão arterial; EF - exercício físico; HPE - hipotensão pós-exercício; NT - normotenso; LT - limítrofe; HT 1 - hipertenso estágio 1; HT - hipertenso; A - ativo; S - sedentário; LA - limiar anaeróbio; NI - não informado; FC máx - frequência cardíaca máxima; FCres - frequência cardíaca de reserva; $\rightarrow$ : não houve HPE significativa pós-

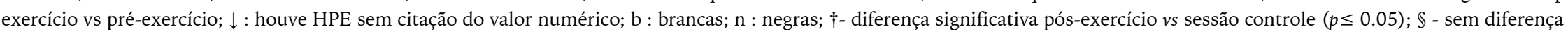
significativa entre os protocolos de durações ou intensidades distintas; * - com diferença significativa entre os grupos II/ID (com polimorfismo ACE) e DD (sem polimorfismo ACE). Para

os demais valores: sinais positivos $(+)$ houve elevação e sinais negativos $(-)$ houve redução significativa dos valores pressóricos pós-exercício vs pré-exercício ( $p \leq 0.05)$. 
2007; MacDonald et al., 2000), diastólicas (Forjaz, Santaella, et al., 1998; MacDonald et al., 2000; Mota et al., 2009) e média (Forjaz, Santaella, et al., 1998; MacDonald et al., 2000) foi aos 45 minutos de recuperação (tabela 3 ). Alguns estudos citam que as reduções pressóricas ocorreram durante todo o período de recuperação, na PA sistólica (Cunha et al., 2006; Ferreira et al., 2013; Forjaz, Matsudaira, et al., 1998), diastólica (Ferreira et al., 2013; Forjaz, Matsudaira, et al., 1998; Lizardo et al., 2007) e média (Cunha et al., 2006; Forjaz, Matsudaira, et al., 1998).

Em relação ao sexo da amostra, $40 \%$ dos estudos incluíram homens e mulheres (Forjaz et al., 2000, 2004; Forjaz, Matsudaira, et al., 1998; Forjaz, Santaella, et al., 1998; MacDonald et al., 1999, 2000; Mota et al., 2009; Rossow et al., 2010) sendo que em apenas dois deles (Forjaz et al., 2000; Rossow et al., 2010) houve distinção entre os resultados de HPE de homens e mulheres. Forjaz et al. (2000) demonstrou que em normotensos, a resposta pressórica pós-exercício foi maior nas mulheres do que nos homens. (Rossow et al., 2010). Por outro lado, o estudo realizado por Rossow et al. (2010) concluiu que, independente da intensidade do exercício, a hipotensão pósexercício ocorre de forma semelhante em homens e mulheres normotensos, não havendo influência nem da intensidade do exercício e nem do sexo.

Quando os dados da HPE foram estratificados por nível de atividade física para aqueles estudos que a identificou em sua amostra, observamos que os indivíduos ativos apresentaram maiores diminuições de pressão arterial quando comparados aos sedentários, tanto no grupo de normotensos quanto no de hipertensos. A média das diminuições pressóricas dos hipertensos ativos vs. sedentários foi de $-16 \pm$ 4 vs. $-9 \pm 2 \mathrm{mmHg}$ para a pressão sistólica, $-7 \pm 4$ vs. $-5 \pm 3 \mathrm{mmHg}$ para a pressão diastólica. Já as HPE dos normotensos ativos vs sedentários foram $-10 \pm 4$ vs. $-8 \pm 1 \mathrm{mmHg}$ para a pressão sistólica e $-5 \pm 1$ vs. $-4 \pm 1$ mmHg para a pressão diastólica.
Três dos artigos selecionados verificaram a influência dos níveis pressóricos iniciais sobre a magnitude da HPE (Casonatto et al., 2011; Forjaz et al., 2000; Pescatello et al., 1999). Em dois deles, os indivíduos com maiores níveis de PA iniciais apresentaram a magnitude da HPE mais significativa (Casonatto et al., 2011; Forjaz et al., 2000). No outro estudo (Pescatello et al., 1999), analisando uma amostra de mulheres pré-menopáusicas, foi demonstrado que 40 minutos de exercícios de intensidade moderada diminuíram as pressões sistólicas, diastólicas e médias em hipertensas, o que não ocorreu nas normotensas. Alguns autores afirmam inconsistência na ocorrência da HPE nos normotensos (Floras \& Wesche, 1992; MacDonald, 2002; Pescatello et al., 1999) outros (Cardoso Jr et al., 2010; Somers, Conway, Coats, Isea, \& Sleight, 1991) citam que uma sessão de exercício agudo pode promover a redução da pressão arterial clínica durante o período pós-exercício tanto em indivíduos hipertensos quanto em normotensos. Essa informação concorda com um dos artigos selecionado nesta revisão, cuja magnitude HPE sistólica em normotensos foi semelhante aquela observada nos hipertensos (Jones et al., 2007). Apesar da dúvida sobre a ocorrência de HPE em normotensos, há um ponto em comum entre os dados encontrados no presente estudo e outras revisões que abordam este tema (Casonatto \& Polito, 2009; Kenney \& Seals, 1993; MacDonald, 2002) em que todos afirmam que, indivíduos hipertensos geralmente apresentam maiores reduções pressóricas que os normotensos nos momentos pós-exercício.

Seis artigos selecionados verificaram a influência da intensidade do exercício sobre a magnitude da HPE (Casonatto et al., 2011; Ferreira et al., 2013; Forjaz et al., 2004; Forjaz, Matsudaira, et al., 1998; MacDonald et al., 1999; Pescatello et al., 2004). No único deles com indivíduos hipertensos, concluiu-se que o exercício a $40 \%$ do $\mathrm{VO}_{2}$ máximo foi tão eficaz quanto a $60 \%$ para provocar HPE, sendo essa intensidade considerada um estímulo suficiente para promover uma queda significativa da 
PA (Pescatello et al., 2004). Noutros quatro estudos selecionados, foram comparados: exercícios com intensidades de 30, 50 e $80 \%$ do $\mathrm{VO}_{2}$ de pico (Forjaz, Matsudaira, et al., 1998); exercícios com intensidades de 50 e $75 \%$ de $\mathrm{VO}_{2}$ de pico (MacDonald et al., 1999); exercícios com intensidades de $80 \%, 60 \%$ e $40 \%$ de $\mathrm{VO}_{2}$ pico, com a duração das sessões que variavam de 30 a 60 minutos (Casonatto et al., 2011); exercícios com intensidades de 50\% (com duração acima de 30 minutos) e de $70 \%$ (com duração de 30 minutos) da FC de reserva. Nestes não foi detetada influência das diferentes intensidades dos protocolos sob a magnitude da HPE em normotensos. No entanto, outro estudo selecionado (Forjaz et al., 2004) apontou que uma maior HPE é obtida nos normotensos a partir de exercícios de maiores intensidades. Assim, podemos conjeturar que não há um consenso sobre a intensidade do exercício e sua influência na HPE em normotensos entre os estudos selecionados, mas a sua maioria indica que este fenómeno independe desta variável.

Quatro artigos selecionados verificaram a influência da duração do exercício sobre a magnitude da HPE em normotensos (Casonatto et al., 2011; Christofaro et al., 2008; Forjaz, Santaella, et al., 1998; MacDonald et al., 2000) e dois deles concluíram que a duração do exercício parece influenciar na magnitude da resposta hipotensiva (Christofaro et al., 2008; Forjaz, Santaella, et al., 1998). Sendo os exercícios de curta e longa duração capazes de promover HPE (Christofaro et al., 2008), porém, o de maior duração, evocando uma HPE maior e mais prolongada (Forjaz, Santaella, et al., 1998). Opostamente aos estudos anteriores (MacDonald et al., 2000), foi demonstrado que a duração do exercício não desempenha um papel importante na determinação da ocorrência ou da magnitude da HPE, uma vez que ela pode ocorrer de forma significativa após exercícios de variadas durações. Diferentemente desses achados, um artigo (Casonatto et al., 2011) verificou que independente da duração da sessão do exercício, sujeitos normotensos não apresentaram HPE.

\section{CONCLUSÕES}

As variedades e as qualidades metodológicas dos artigos selecionados e avaliados não permitiram conclusões definitivas sobre o tema da HPE. Contudo, de acordo com as observações individuais dos artigos podemos afirmar que as menores quedas pressóricas ocorridas foram de $-4 \mathrm{mmHg}$ para a sistólica (Christofaro et al., 2008; Santana et al., 2011), -2 mmHg para a diastólica (Forjaz et al., 2000; Pescatello et al., 1999), $-1 \mathrm{mmHg}$ (Santana et al., 2011) e $-2 \mathrm{mmHg}$ para a média (Forjaz et al., 2000). Já as maiores magnitudes da HPE ocorreram em estudo com amostra de hipertensos (Cunha et al., 2006), sendo de19 e $-18 \mathrm{mmHg}$ para a sistólica e de-13 e -10 mmHg para a média. Para a diastólica a redução pressórica foi de -9 (Cunha et al., 2006) e $-8 \mathrm{mmHg}$ (Pescatello et al., 2003).

Neste estudo também observamos que a magnitude da HPE depende de vários fatores como as características do exercício realizado, sua intensidade e duração, e o perfil da amostra, sendo algumas delas o sexo, estágios pressóricos iniciais, presença ou não da HAS e o nível de atividade física. Não foi observada concordância entre os resultados obtidos nos estudos selecionados sobre a influência da intensidade e duração do exercício sobre a HPE, pois, em alguns se observou diferenças significativas (Christofaro et al., 2008; Forjaz et al., 2004; Forjaz, Santaella, et al., 1998), ao contrário de outros em que não foram encontradas diferenças significativas nas reduções pressóricas (Casonatto et al., 2011; Ferreira et al., 2013; Forjaz, Matsudaira, et al., 1998; MacDonald et al., 1999, 2000; Pescatello et al., 2004). Com relação à presença de homens e mulheres na amostra dos estudos avaliados, entendemos que a presença do sexo nas pesquisas e suas diferenças não foi um fator analisado, deste modo, não podemos concluir sobre a sua real interferência na HPE. Com relação aos níveis pressóricos iniciais verificamos que os hipertensos possuem uma magnitude mais 
significativa da HPE em relação aos normotensos. Ao se comparar os indivíduos ativos com os sedentários, os ativos foram mais beneficiados por esta redução.

As investigações científicas a respeito da HPE são necessárias a fim de enriquecer as informações a respeito deste tema de grande importância clínica, como também para esclarecer contradições ainda existentes sobre os fatores que influenciam a ocorrência das quedas pressóricas após exercício físico.

\section{Agradecimentos:}

Nada a declarar.

\section{Conflito de Interesses:}

Nada a declarar.

Financiamento:

Nada a declarar.

\section{REFERÊNCIAS}

Altman, D. G., \& Dore, C. J. (1990). Randomisation and baseline comparisons in clinical trials. The Lancet, 335(8682), 149-153. doi: 10.1016/01406736(90)90014-V

Cal Abad, C. C., da Silva, R. S., Mostarda, C., da Silva, I. C. d. M., \& Irigoyen, M. C. (2010). Efeito do exercício aeróbico e resistido no controle autonômico e nas variáveis hemodinâmicas de jovens saudáveis. Revista Brasileira de Educação Física e Esporte, 24, 535-544.

Cardoso Jr, C. G., Gomides, R. S., Queiroz, A. C., Pinto, L. G., da Silveira Lobo, F., Tinucci, T., ... Forjaz, C. L. M. (2010). Acute and chronic effects of aerobic and resistance exercise on ambulatory blood pressure. Clinics, 65(3), 317325. doi: 10.1590/S1807-59322010000300013

Casonatto, J., \& Polito, M. (2009). Hipotensão pósexercício aeróbio: Uma revisão sistemática. Revista Brasileira de Medicina do Esporte, 15, 151157. doi: 10.1590/S1517-86922009000200014

Casonatto, J., Tinucci, T., Dourado, A. C., \& Polito, M. D. (2011). Cardiovascular and autonomic responses after exercise sessions with different intensities and durations. Clinics, 66(3), 453458. doi: 10.1590/S1807-59322011000300016

Chan, A. W., \& Altman, D. G. (2005). Epidemiology and reporting of randomised trials published in PubMed journals. The Lancet, 365(9465), 11591162. doi: 10.1016/S0140-6736(05)71879-1

Christofaro, D. G., Casonatto, J., Fernandes, R. A., Cucato, G. G., Gonçalves, C. G. S., Ramos de Oliveira, A., \& Polito, M. D. (2008). Efeito da duração do exercício aeróbio sobre as respostas hipotensivas agudas pós-exercício. Revista da SOCERJ, 21 (6), 404-408.

Corazza, D. I., Gobbi, S. Z., Zago, A. S., \& Costa, J. L. R. (2003). Hipotensão pós-exercício: Comparação do efeito agudo do exercício aeróbio em mulheres normotensas e hipertensas limítrofes, da terceira idade adulta. Revista Brasileira de Atividade Física \& Saúde, 8(2), 28-34.

Cunha, G., Rios, A., Moreno, J., Braga, P., Campbell, C., Simões, H., \& Denadai, M. (2006). Hipotensão pós-exercício em hipertensos submetidos ao exercício aeróbio de intensidades variadas e exercício de intensidade constante. Revista Brasileira de Medicina do Esporte, 12 (6), 313-317. doi: 10.1590/S1517-86922006000600003

Ferreira, A. P., Campos, B. R. M., Rodrigues Junior, E., Puga, G. M., \& Brito, C. J. (2013). Effects of aerobic and resistance exercise intensities on 24-hours blood pressure in normotensive women. Motriz: Revista de educação física, 19, 681687. doi: 10.1590/S1980-65742013000400003

Ferreira, A. P., Ferreira, C. B., Campos, B. R. M., Samy, G. C. P., de Morais, P. P. (2011). Efeito de diferentes intensidades de exercício aeróbio na resposta pressórica de 24 horas em mulheres normotensas. Journal of the Health Sciences Institute, 29(1), 62-66.

Floras, J. S., \& Wesche, J. (1992). Haemodynamic contributions to post-exercise hypotension in young adults with hypertension and rapid resting heart rates. Journal of Human Hypertension, 6(4), 265-269.

Forjaz, C. L., Cardoso, C. G., Rezk, C. C., Santaella, D. F. \& Tinucci, T. (2004). Postexercise hypotension and hemodynamics: The role of exercise intensity. Journal of Sports Medicine and Physical Fitness, 44(1), 54-62.

Forjaz, C. L. M., Matsudaira, Y., Rodrigues, F. B., Nunes, N., \& Negrão, C. E. (1998). Postexercise changes in blood pressure, heart rate and rate pressure product at different exercise intensities in normotensive humans. Brazilian 
Journal of Medical and Biological Research, 31 (10), 1247-1255. doi: 10.1590/S0100-879X1998001 000003

Forjaz, C. L. M., Santaella, D. F., Rezende, L. O., Barretto, A. C. P., \& Negrão, C. E. (1998). A duração do exercício determina a magnitude e a duração da hipotensão pós-exercício. Arquivos Brasileiros de Cardiologia, 70(2), 99-104. doi: 10.1590/S0066-782X1998000200006

Forjaz C. L. M., Tinucci, T., Ortega, K. C., Santaella, D. F., Mion Jr, D., \& Negrão, C. E. (2000). Factors affecting post-exercise hypotension in normotensive and hypertensive humans. Blood Pressure Monitoring, 5(5-6), 255-262.

Hagberg, J. M., Park, J. J., \& Brown, M. D. (2000). The role of exercise training in the treatment of hypertension: An update. Sports Medicine, 30(3), 193-206. doi: 10.2165/00007256-20003003000004

Jadad, A. R., Moore, R. A., Carrol, D., Jenkinson, C., Reynolds, J. M., Gavaghan, D. J., \& McQuay, H. J. (1996). Assessing the quality of reports of randomized clinical trials: Is blinding necessary? Controlled Clinical Trials, 17, 1-12. doi: 10.1016/0197-2456(95)00134-4

Jones, H., George, K., Edwards, B., \& Atkinson, G. (2007). Is the magnitude of acute post-exercise hypotension mediated by exercise intensity or total work done? European Journal of Applied Physiology, 102, 33-40. doi: 10.1007/s00421007-0562-0

Kenney, M. J., \& Seals, D. R. (1993). Postexercise hypotension: Key features, mechanisms, and clinical significance. Hypertension, 22, 653-664.

Lizardo, J. H. F., Modesto, L. K., Campbell, C. S. G., \& Simões, H. G. (2007). Hipotensão pósexercício: Comparação entre diferentes intensidades de exercício em esteira ergométrica e cicloergômetro. Revista Brasileira de Cineantropometria e Desempenho Humano, 9(2), 115-120.

MacDonald, J. R. (2002). Potential causes, mechanisms, and implications of post exercise hypotension. Journal of Human Hypertension, 16(4), 225-236. doi: 10.1038/sj/jhh/1001377

MacDonald, J. R., MacDougall, J. D., \& Hogben, C. D. (1999). The effects of exercise intensity on post exercise hypotension. Journal of Human $\mathrm{Hy}$ pertension, 13(8), 527-531.

MacDonald, J. R., MacDougall, J. D., \& Hogben, C. D. (2000). The effects of exercise duration on post-exercise hypotension. Journal of Human $\mathrm{Hy}$ pertension, 14(2), 125-129.
Moher, D., Hopewell, S., Schulz, K. F., Montori, V., Gøtzsche, P. C., Devereaux, P. J., ... Altman, D. G. (2010). Research Methods \& Reporting: CONSORT 2010 Explanation and Elaboration: updated guidelines for reporting parallel group randomised trials. British Medical Journal, 340, c869. doi: 10.1136/bmj.c869

Moher, D., Jadad, A. R., Nichol, G., Penman, M., Tugwell, P., \& Walsh, S. (1995). Assessing the quality of randomized controlled trials: An annotated bibliography of scales and checklists. Controlled Clinical Trials, 16(1), 62-73.

Mota, M. R., Pardono, E., Lima, L. C. J., Arsa, G., Bottaro, M., Campbell, C. S. G., \& Simões, H. G. (2009). Effects of treadmill running and resistance exercises on lowering blood pressure during the daily work of hypertensive subjects. The Journal of Strength \& Conditioning Research, 23(8), 2331-2338. doi: 10.1519/JSC.0b013e31 81 bac 418

Pescatello, L. S., Bairos, L., VanHeest, J. L., Maresh, C. M., Rodriguez, N. R., Moyna, N. M., ... Krueger, L. (2003). Postexercise hypotension differs between white and black women. American Heart Journal, 145(2), 364-370. doi: 10.1067/mhj.2003.107

Pescatello, L. S., Guidry, M. A., Blanchard, B. E., Kerr, A., Taylor, A. L., Johnson, A. N., ... Thompson, P. D. (2004). Exercise intensity alters postexercise hypotension. Journal of Hypertension, 22 (10), 1881-1888.

Pescatello, L. S., Miller, B., Danias, P. G., Werner, M., Hess, M., Baker, C., \& Jane De Souza, M. (1999). Dynamic exercise normalizes resting blood pressure in mildly hypertensive premenopausal women. American Heart Journal, 138(5 Pt1), 916-921. doi: 10.1016/S0002-8703(99) 70 017-7

Polito, M. D., Simão, R., Saccomani, M. G., \& Casonatto, J. (2009). Influência de uma sessão de exercício aeróbio e resistido sobre a hipotensão pós-esforço em hipertensos. Revista da SOCERJ, 22(5), 330-334.

Rech, J. B. S., Nóbrega, L., \& Lemos, A. (2010). Compressão pneumática no tratamento de linfedema pós-mastectomia: Revisão sistemática. Revista Brasileira de Cancerologia, 56 (4), 483-491.

Rossow, L., Yan, H., Fahs, C. A., Ranadive, S. M., Agiovlasitis, S., Wilund, K. R., ... Fernhall, B. (2010). Postexercise hypotension in an endurance-trained population of men and women following high-intensity interval and steady-state 
cycling. American Journal of Hypertension, 23(4), 358-367. doi: 10.1038/ajh.2009.269

Santana, H. A. P., Moreira, S. R., Asano, R. Y., Sales, M. M., Cordova, C., Campbell, C. S., ... Simoes, H. G. (2013). Exercise intensity modulates nitric oxide and blood pressure responses in hypertensive older women. Aging Clinical and Experimental Research, 25(1), 43-48. doi: 10.1007/ s40520-013-0017-x

Santana, H. A. P., Moreira, S. R., Neto, W. B., Silva, C. B., Sales, M. M., Oliveira, V. N., ... Campbell, C. S. G. (2011). The higher exercise inten- sity and the presence of allele I of ACE gene elicit a higher post-exercise blood pressure reduction and nitric oxide release in elderly women: an experimental study. BMC Cardiovascular disorders, 11(1), 71. doi: 10.1186/14712261-11-71

Somers, V. K., Conway, J., Coats, A., Isea, J., \& Sleight, P. (1991). Postexercise hypotension is not sustained in normal and hypertensive humans. Hypertension, 18(2), 211-215. doi: 10.1161/ 01.HYP.18.2.211

Todo o conteúdo da revista Motricidade está licenciado sob a Creative Commons, exceto quando especificado em contrário e nos conteúdos retirados de outras fontes bibliográficas. 